\title{
Lipoabdominoplastia con desinserción umbilical y despegamiento ahdominal reducido
}

\section{Lipoabdominoplasty with umbilical detachment and reduced abdominal undermining}

\author{
Cido Carvalho, F. A. M.*, Vieira da Silva J r., V.**, Alencar Moreira, A.***
}

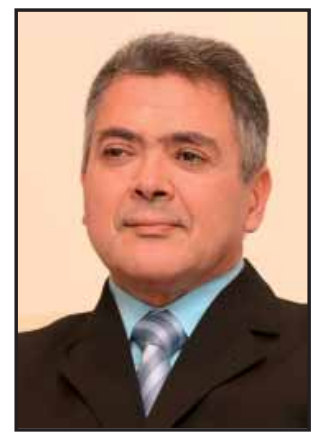

Cido Carvalho, F. A. M.

\section{Resumen}

Presentamos una combinación de procedimientos quirúrgicos para el tratamiento del exceso de volumen abdominal con flacidez moderada, utilizando una miniabdominoplastia con despegamiento y desinserción umbilical, asociada a liposucción. La indicación de la técnica se restringe a aquellos casos en los que la liposucción aislada provocaría un agravamiento de la flacidez, mientras que una abdominoplastia clásica implicaría una resección exagerada de piel o la necesidad de dejar una pequeña cicatriz vertical. Una miniabdominplastia trataría solamente la flacidez del abdomen inferior, dejando un exceso de piel en la región supraumbilical.

La combinación de liposucción amplia y despegamiento controlado del área central del abdomen, asociada a una desinserción umbilical y su posterior reinserción unos 2 a $4 \mathrm{~cm}$ más abajo, nos permite lograr una completa plicatura de los rectos abdominales, con una buena resección del exceso cutáneo del abdomen inferior, además de un tratamiento adecuado de la flacidez moderada del abdomen superior.

\section{Palahras clave Lipoabdominoplastia. Dermolipectomía abdominal. Desinserción umbilical. \\ Código numérico 5311-534}

$\begin{array}{ll}\text { Key words } & \text { Lipoabdominoplasty. Abdominal dermo- } \\ & \text { lictomy. Navel detachment. } \\ \text { Numeral Gode } & 5311-534\end{array}$

Numeral Gode
We present a combination of procedures for the treatment of the abdominal volume excess with moderate skin excess, using lipoabdominoplasty with detachment of the navel. Indications are restricted to those cases where the isolated suction-assisted lipoctomy (SAL) would take to the worsening of the skin flaccidity, while the full abdominoplasty would imply in exaggerated excision of the skin or presence of small vertical scar. The miniabdominoplasty more frequently used would only treat the flaccidity about the inferior abdomen, leaving skin excess in the upper abdomen area. The combination of wide suctionassisted lipectomy with undermining in the central area of the abdomen, with umbilical detachment and its posterior new implantation about 2 to $5 \mathrm{~cm}$ lower, allows a complete plication of the abdominal rectums, with good excision of the skin excess of the inferior abdomen, besides the treatment of the moderate flaccidity of the superior abdomen. $\begin{array}{ll}* & \text { Jefe de Senvicio. } \\ \text { * } & \text { Médico Especialista. } \\ \text { *** } & \text { Médico Residente. }\end{array}$

Servicio de Cirugía Plástica do Instituto Dr. J osé Frota, Fortaleza, Ceará, Brasil. 


\section{Introducción}

Las alteraciones estéticas del abdomen superior y de la región infraumbilical pueden aparecer de forma aislada o combinadas con un aumento del volumen abdominal y la presencia de flacidez cutánea. Existen varias clasificaciones para el diagnóstico y tratamiento de las diversas patologías de la morfología abdominal. Matarasso (1), clasifica en 4 categorías los diversos tipos de abdomen, mientras que Bozola y Psillakis (2) destacan la existencia de 5 diferentes niveles de deformidad, a la vez que ofrecen un tipo de tratamiento para cada una de ellas.

Los pacientes que presentan un exceso de grasa, pero que no tienen flacidez, tienen una indicación específica de liposucción aislada, mientras que el tratamiento de elección en aquellos casos en que hay aumento de volumen asociado a flacidez cutánea, tienen indicación de abdominoplastia clásica. Aquellos casos en los que el exceso de volumen y la flacidez están restringidos al abdomen inferior, pueden beneficiarse de una miniabdominoplastia con resultados satisfactorios. Sin embargo, hay casos en los que el paciente presenta diástasis de rectos abdominales, aumento del volumen graso y flacidez moderada también en el abdomen superior, pero sin indicación de abdominoplastia clásica, lo que supone limitaciones en el tratamiento $(1,3-5)$.

La liposucción de todo el abdomen, con incisión reducida y despegamiento abdominal central amplio desinsertando el ombligo y manteniéndolo unido al colgajo, nos permite una corrección de la diástasis de los rectos, una buena resección del exceso de piel inferior, además de un tratamiento adecuado de la flacidez del abdomen superior.

\section{Material y método}

Hemos intervenido 58 pacientes durante el periodo comprendido entre 1994 y 2007, del sexo femenino y con una edad media de 41 años (de 23 a 52 años), todas mediante anestesia epidural.

La técnica empleada fue una combinación de procedimientos consagrados en la literatura científica al respecto; hacemos una marcación previa de la porción de piel a resecar en el abdomen inferior. Inicialmente se realiza lipoaspiración del dorso, de la cintura y de toda la región anterior del abdomen. En el área central supraumbilical, se realiza liposucción en el plano superficial y en el profundo, a fin de lograr una mayor definición de los músculos rectos abdominales. En el resto del abdomen, la liposucción se hizo en un plano profundo a fin de presevar al máximo la irrigación cutánea una vez que hagamos el despegamiento de un área considerable (Fig. 1). Tras la liposucción (Cuadro 1), realizamos una incisión baja en la región pubiana, curva, de longitud que varió entre los 13 y los $18 \mathrm{~cm}$ (Cuadro 2). El despegamiento se llevó a cabo en toda la región que iba a ser resecada, extendiéndose superiormente en la región media abdominal hasta aproximadamente $5 \mathrm{~cm}$ del borde lateral de los rectos, seccionando el ombligo de su base, y manteniéndolo fijo al colgajo abdominal (Fig. 1). Esto permitió corregir una eventual diástasis mediante plicatura de los rectos abdominales desde xifoides a pubis (Fig. 2). Tras la plicatura, se reinsertó el ombligo sobre la aponeurosis, aproximadamentes 2 a $5 \mathrm{~cm}$ más abajo de su posición original, con el propósito de tratar también la flacidez de la piel del abdomen superior (Cuadro 3). Empleamos puntos de nylon 3-0 para configurar fácilmente la longitud, tamaño y profundi-

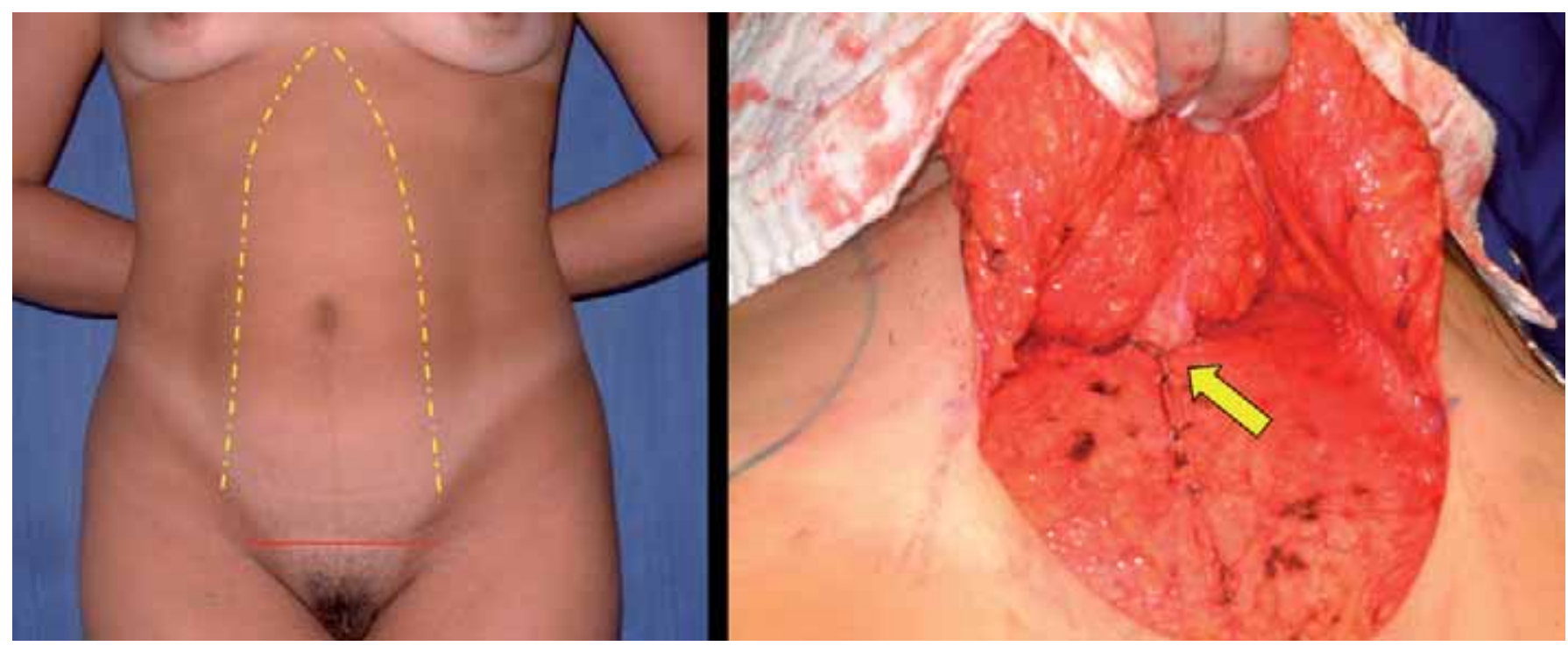

Fig. 1. Izquierda: área de despegamiento marcada con la línea de trazos en color amarillo y la incisión marcada en rojo. Derecha: desinserción del ombligo (flecha).

Esquerdo: Área de descolamento marcado com linha amarela tracejada e incisão em vermelho. Direita: Desinserção do umbigo (seta). 


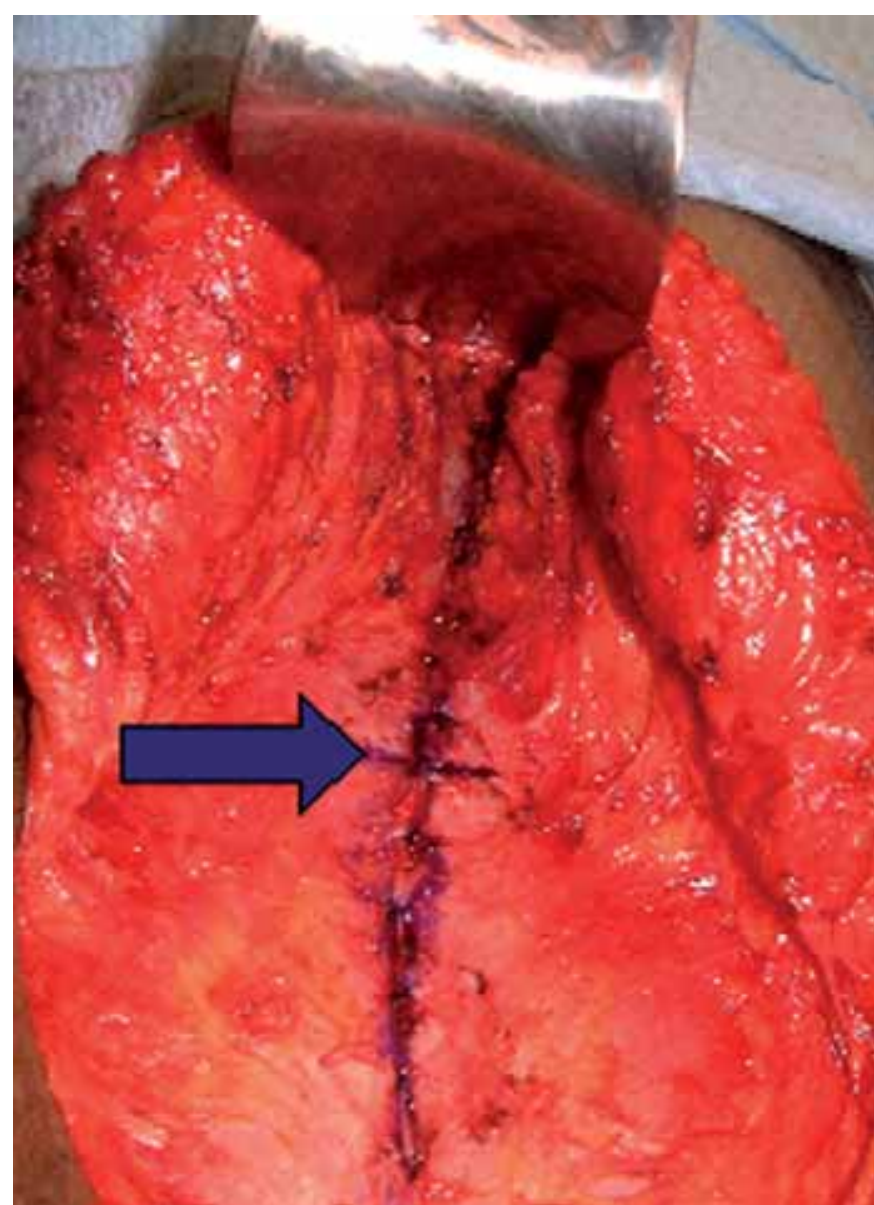

Fig. 2. Plicatura de los rectos abdominales y marcación de la nueva posición umbilical (flecha).

Plicatura dos retos e marcação da nova posição do umbigo (seta).

dad del nuevo ombligo. Excepcionalmente, en casos de mayor flacidez del abdomen superior y ombligo alto, podemos llevar a cabo la reinserción umbilical hasta $5 \mathrm{~cm}$ más abajo (Fig. 2).

A continuación, practicamos la resección del exceso de piel previamente marcado, pudiendo incluso ampliar esa resección por haberse producido tras la liposucción y el despegamiento una mayor flacidez de la esperada. La porción de piel resecada del abdomen inferior varió en nuestros casos entre los 8 y 11 cm (Cuadro 4) (Fig. 3).

El cierre de la herida quirúrgica se realizó con sutura intradérmica, colocándose drenajes de aspiración y presoterapia con banda elástica que mantendremos durante 2 meses.

\section{Resultados}

En el periodo 1994 a 2007 intervenimos 58 pacientes de sexo femenino, con una media de edad de 41 años (de 23 a 52 años), todas ellas bajo anestesia epidural. De ellas, 48 presentaban aumento de volumen abdominal y flacidez moderada del abdomen superior; en 2 casos había flacidez más acentuada y 3 pacientes ya habían sido sometidas a abdominoplastia
Cuadro1. Volumen aspirado durante la lipoabdominoplastia:

\begin{tabular}{lr} 
Volumen & $\mathbf{n}$ \\
1000 a $1500 \mathrm{ml}$ & 22 \\
1500 a $2000 \mathrm{ml}$ & 25 \\
2000 a $2200 \mathrm{ml}$ & 11 \\
Total & 58 \\
\hline
\end{tabular}

Cuadro 2. Extensión de la incisión de lipoabdominoplastia:

\begin{tabular}{lr} 
Tamaño & $\mathbf{n}$ \\
13 a $14 \mathrm{~cm}$ & 22 \\
14 a $15 \mathrm{~cm}$ & 25 \\
15 a $16 \mathrm{~cm}$ & 07 \\
16 a $17 \mathrm{~cm}$ & 03 \\
17 a $18 \mathrm{~cm}$ & 01 \\
Total & 58 \\
\hline
\end{tabular}

Cuadro 3. Altura de reinserción del ombligo tras la rescción del exceso de piel:

\begin{tabular}{lr} 
Reinserción del ombligo & n \\
$3 \mathrm{~cm}$ más bajo & 39 \\
$4 \mathrm{~cm}$ más bajo & 17 \\
$5 \mathrm{~cm}$ más bajo & 02 \\
Total & 58 \\
\hline
\end{tabular}

Cuadro 4. Extensión de la resección del exceso de piel tras el despegamiento del colgajo abdominal (sin aplicar tracción):

\begin{tabular}{lr} 
Resección de piel & n \\
8 a $9 \mathrm{~cm}$ & 32 \\
9 a $10 \mathrm{~cm}$ & 18 \\
10 a $11 \mathrm{~cm}$ & 08 \\
Total & 58 \\
\hline
\end{tabular}

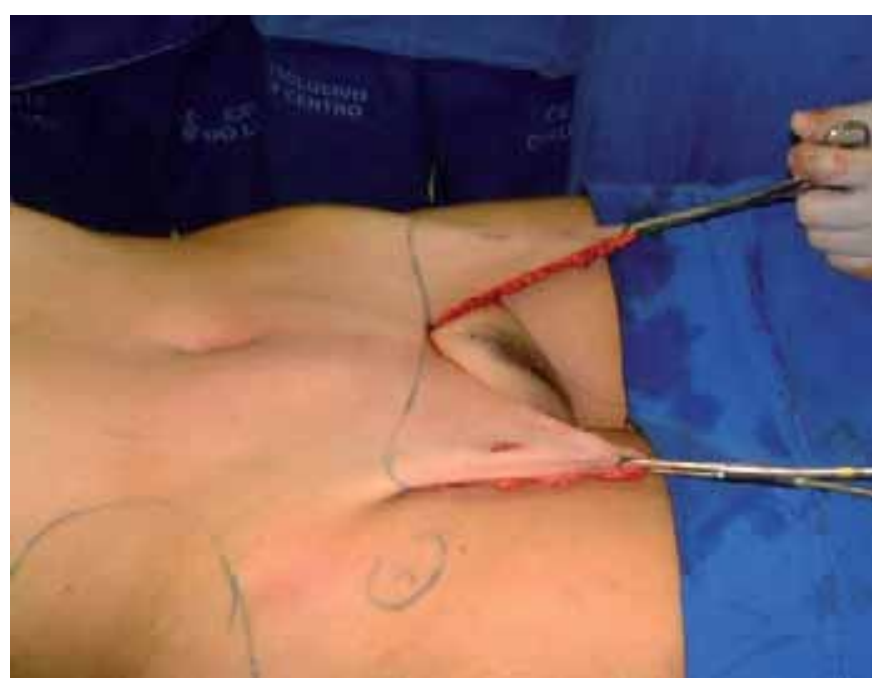

Fig. 3. Imagen del colgajo despegado y marcación para la resección de $10 \mathrm{~cm}$ de piel.

Tração do retalho descolado e marcação para ressecção de $10 \mathrm{~cm}$ de pele. 


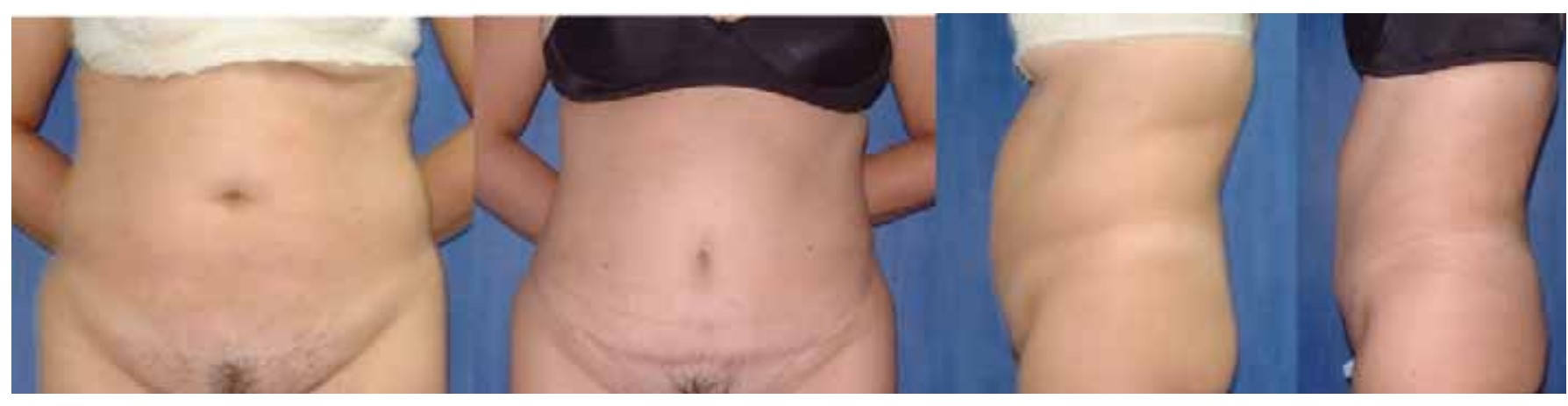

Fig. 4. Pre y postoperatorio en vista frontal y perfil izquierdo. La paciente presentaba un abdomen superior muy corto. El ombligo fue reinsertado $4 \mathrm{~cm}$ más abajo de su posición original.

Pré e pós-operatório numa vista frontal e de perfil esquerdo. A paciente apresentava abdome superior muito curto. 0 umbigo foi reinserido 4 cm abaixo da posição original.

Cuadro 5. Distribución preoperatoria de las características del abdomen de las pacientes:

Características del abdómen

n

Aumento de volumen total y

flacidez moderada del abdomen superior

Aumento de volume y flacidez

más acentuada del abdomen superior

Abdominoplastia clásica anterior

Miniabdominoplastia anterior

Total

03

05

58

Cuadro 6. Complicaciones:

\section{Complicaciones}

Presencia de alguna flacidez

en el abdomen superior

Cicatriz alargada

Ondulaciones en el área aspirada

Total
02

01

02

05 clásica pero, a pesar de un resultado satisfactorio en el abdomen inferior, presentaban flacidez residual y exceso de grasa en el abdomen superior. Cinco pacientes ya habían sido sometidas a miniabdominoplastia, manteniendo flacidez y exceso de volumen en el abdomen superior (Cuadro 5). Todas las pacientes fueron intervenidas empleando la misma combinación de procedimientos quirúrgicos descrita en el presente artículo.

Los resultados fueron satisfactorios en todos los casos en los que había exceso de volumen abdominal y flacidez del abdomen inferior con moderada flacidez del abdomen superior. En 2 pacientes la flacidez del abdomen superior sugería indicación de abdominoplastia completa, pero las pacientes manifestaron su intención de tener embarazos en el futuro por lo que no aceptaron esta indicación y en ellas quedó alguna flacidez residual en la parte superior. En las restantes pacientes, las complicaciones fueron raras y consistieron en pequeñas irregularidades y discretas alteraciones cicatricilaes que fueron corregidas posteriormente con procedimientos ambulatorios (Cuadro 6) (Fig. 4-6).

\section{Discusión}

Las alteraciones estéticas de la región abdominal pueden ser corregidas fácilmente mediante liposucción aislada cuando no hay flacidez abdominal. También el abordaje es fácil cuando se trata de pacientes con gran volumen graso y mucha flacidez, mediante abdominoplastia clásica o mediante miniabdomino-
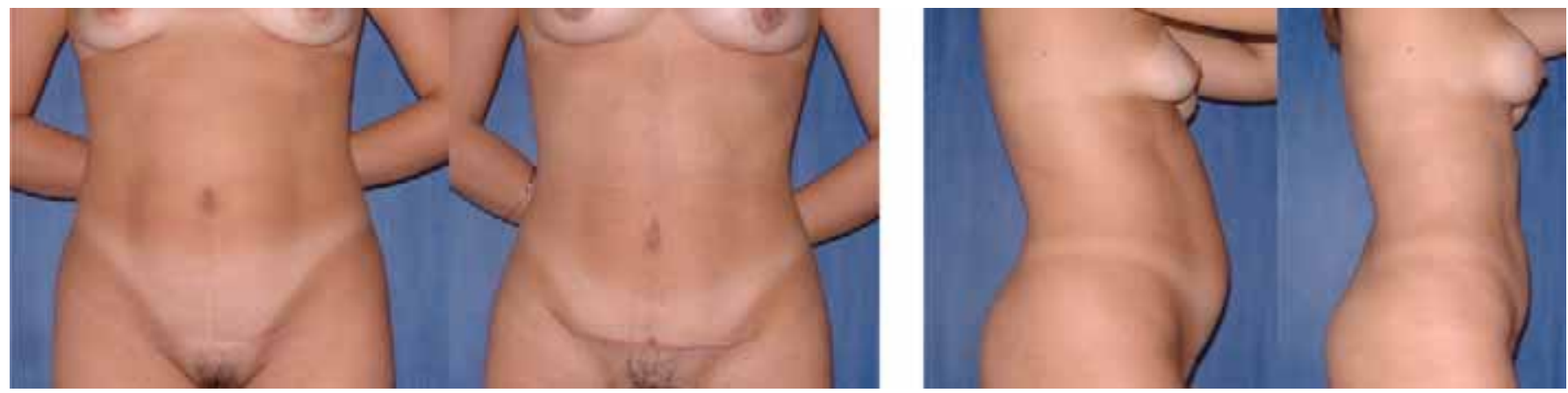

Fig. 5. Pre y postoperatorio en vista frontal y perfil derecho. La paciente no presentaba ombligo alto, pero tenía indicación de cirugía. La cicatriz abdominal fue trasladada $2,5 \mathrm{~cm}$ más abajo de su posición original.

Pré e pós-operatório numa vista frontal e de perfil direito. A paciente não apresentava umbigo alto mas teve boa indicação de cirurgia. A cicatriz abdominal foi deslocada $2,5 \mathrm{~cm}$ abaixo da posição original. 


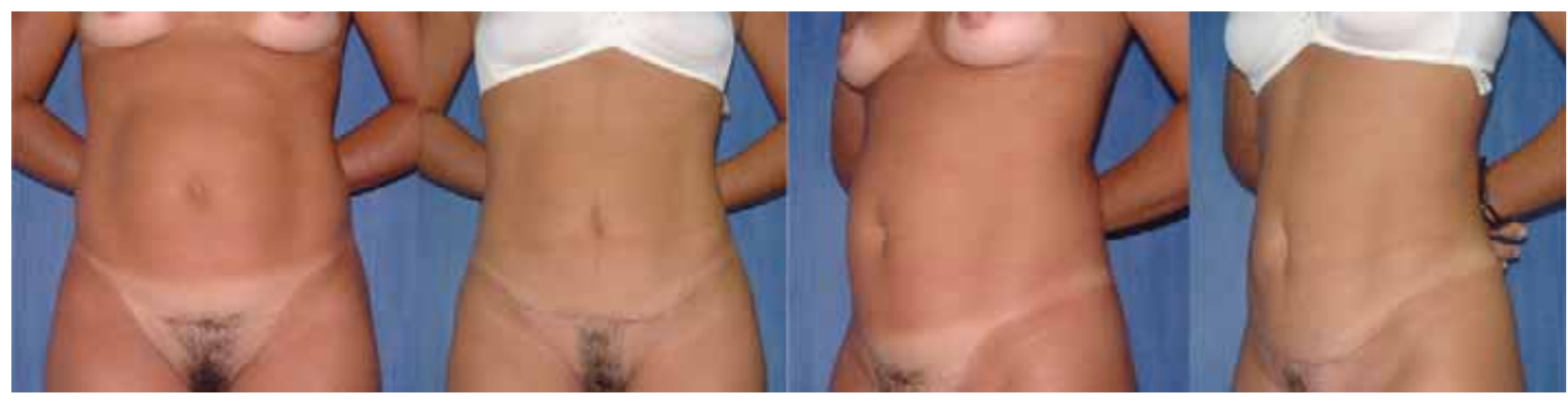

Fig. 6. Pre y postoperatorio en vista frontal y oblicua izquierda. La paciente presentaba flacidez moderada de abdomen superior y una gestación anterior.

Pré e pós-operatório numa vista frontal e oblíquo esquerdo. Paciente apresentava flacidez moderada de abdomen superior e uma gestação anterior.

plastia convencional cuando la deformidad se restringe a la región infraumbilical (1,3-5). Mientras tanto, la solución en los casos de flacidez moderada del abdomen superior con ombligo excesivamente alto, son más complicados de resolver, siendo necesarias para ello medidas no convencionales.

El tratamiento de este tipo de casos es un tema ampliamente estudiado. Hackme (6) publicó un detallado estudio sobre la evolución de la técnicas para la corrección de los excesos abdominales. El tratamiento de los casos de exceso de volumen graso en pacientes sin flacidez cutánea, tuvo en los trabajos de Illouz (7) una gran evolución mediante el empleo de la liposucción. Callia (8) realizó un minucioso estudio sobre el tratamiento del abdomen péndulo de gran volumen, mientras que Wilkinson y Swartz (9) y de Greminger (10) introdujeron importantes conceptos en el tratamiento del abdomen inferior.

La cicatriz umbilical también ha sido muy estudiada y están descritos diseños muy variados de abordaje externo, así como la liberación del ombligo de su implantación aponeurótica y su posterior reinserción, descrita por Callia (8) y también utilizada por Avelar (11), que lleva a cabo su implantación unos $2 \mathrm{~cm}$ más abajo de su posición original.

Tras una amplia utlización de la liposucción, diversos estudios demostraron la posibilidad de su uso asociado a abdominoplastia. Matarasso $(3,4)$ emplea liposucción en todos los tipos de abdominplastia, pero restringiendo su uso sólo al área que no va a ser despegada. Valiosos estudios anatómicos como los de Huger (12) permitieron que se realizaran procedimientos cada vez más seguros en la abdominoplastia. El despegamiento limitado hizo que la técnica fuera más segura, pues preserva la vascularización del colgajo $(4,13)$. Saldaña (13) y Graf (5) defienden que la liposucción de la región surpaumbilical puede comprometer el colgajo, pero nosotros no hemos tenido ningún signo de sufrimiento en la liposucción de esa área.

\section{Conclusiones}

La técnica empleada es válida, puesto que permite el tratamiento de la flacidez moderada del abdomen superior e inferior con una incisión de un tamaño aceptable. El despegamiento reducido hace viable la realización de una liposucción de volumen considerable. La ausencia de cicatriz externa umbilical evita estigmas, dejando un ombligo de aspecto estético natural y las complicaciones, por lo general, son relativamente raras, semejantes a las que podemos encontrar en cualquier abdominoplastia convencional.

\section{Dirección del autor}

Dr. Francisco de Asis Montenegro Cido Carvalho

Rua Ana Bilhar 44/202- Miereles.

Fortaleza-CE- Brasil

e-mail: drcidocarvalho@yahoo.com.br

\section{Bibliografía}

1. Matarasso, A: "Abdominoplasty: a system of classification and treatment for combined abdominoplasty and suction-assisted lipectomy". Aesth. Plast. Surg. 1991, 15: 111.

2. Bozola, A. R., and Psillakis, J. M.: "Abdominoplasty: A new concept and classification for treatment". Plast. Reconstr. Sur. 1988, 82: 983.

3. Matarasso, A.: "Classification and patient selection in abdominoplasty". Operative Techniques in Plastic and Reconstructive Surgery. 1996, 3(1): 7-14.

4. Matarasso, A.: "Liposuction as an adjunct to full abdominoplasty revisited". Plast. Reconst. Surg. 2000, 106: 1197.

5. Graf, R., de Araujo, L.R., Rippel, R., Neto, L.G., Pace, D.T., Cruz, G.A.: "Lipoabdominoplasty: liposuction with reduced undermining and traditional abdominal skin flap resection". Aesth. Plast. Surg. 2006, 30(1): 1 .

6. Hakme, F.: "Evolução histórica das abdominoplastias e contribuição pessoal”. Ann. Simp. Bras. Abdominoplastia. 1982, 1: 3.

7. Illouz, Y. G.: "Une nouvelle technique pour les lipodystrophies localisées". Rev. Chir. Esthet. 1980, 4: 19.

8. Callia, W.: "Contribuição para o estudo da correção cirúrgica do abdome pêndulo e globoso - Técnica original". Diss. Fac. Med. Univ. São Paulo, 1965.

9. Wilkinson, T. S., Swartz, B. S.: "Individual modifications in body contour surgery: The limited abdominoplasty". Plast. Reconstr. Surg. 1986, 77: 779. 
10. Greminger, R. F.: "The Miniabdominoplasty". Plast. Reconstr. Surg. 1987, 79: 356.

11. Avelar, J. M.: "Abdominoplasty: Methodization of a technique without external umbilical scar”. Aesth. Plast. Surg. 1978, 2: 141.
12. Huger, W. E., Jr.: "The anatomic rationale for abdominal lipectomy". Am. Surg. 1979, 45: 612.

13. Saldanha, O.R., Souza Pinto, E.B., Matos, W.M., Lucon, R.L., Magalhães, F., Bello, E.M.L.: "Lipoabdominoplasty without undermining". Aesth. Surg. J. 2001, 21:518. 


\title{
Lipoabdominoplastia com desinserção umbilical e descolamento reduzido
}

\author{
Cido Carvalho, F. A. M.*, Vieira da Silva J r., V.**, Alencar Moreira, A.*** \\ * Chefe do Serviço e da Residência de Cirugia Plástica. \\ * Especialista do Serviço. \\ * Residente do Serviço. \\ Senviço de Cirugia Plastica, Instituto Dr. J osé Frota, Fortaleza, Ceará, Brazil.
}

Resumo

É apresentada combinação de procedimentos para tratamento do excesso de volume abdominal com flacidez moderada, utilizando lipoabdominoplastia com desinserção do umbigo. A indicação é restrita àqueles casos onde a lipoaspiração isolada resultaria agravamento de flacidez, enquanto a abdominoplastia clássica implicaria em ressecção exagerada de pele ou presença de pequena cicatriz vertical. A miniabdominoplastia mais freqüentemente trataria apenas a flacidez do abdome inferior, restando excesso de pele na região supra-umbilical. Foram operados 58 pacientes entre 1994 e 2007 utilizando esse procedimento. A combinação de lipoaspiração ampla com descolamento dirigido à área central do abdome, associada a desinserção do umbigo e posterior reinserção cerca de 2 a $5 \mathrm{~cm}$ mais inferior permite uma completa plicatura dos retos abdominais, com boa ressecção do excesso de pele no abdome inferior, além de tratamento da flacidez moderada do abdome superior.

\section{Introdução}

As alterações estéticas do abdome superior e da região infraumbilical podem aparecer isoladas ou combinadas, e são relacionadas a aumento de volume abdominal e à presença de flacidez. Há várias classificações para diagnóstico e tratamento de diversas alterações abdominais. Matarasso(1) enquadra em 4 categorias variados tipos de abdome, enquanto Bozola e Psillakis (2) destacam 5 diferentes níveis de deformidades, indicando um tipo de tratamento específico para cada nível.

Pacientes com excesso gorduroso, mas sem flacidez de pele, têm indicação específica de lipoaspiração isolada, enquanto nos casos de volume aumentado e maior flacidez, a abdominoplastia clássica é o tratamento indicado. Nos excessos de volume e flacidez restritos ao abdome inferior, a miniabdominoplastia é realizada com bons resultados. Entretanto, há casos em que há diastase dos retos abdominais, aumento do volume adiposo e flacidez moderada também no abdome superior, mas sem indicação de abdominoplastia clássica, trazendo, com isso, limitações no tratamento (1,3-5).

A lipoaspiração de toda a região abdominal, com incisão reduzida e descolamento central amplo desinserindo o umbigo e mantendo-o preso ao retalho permite correção de diastase dos retos, boa ressecção de excesso de pele inferior, além de tratamento da flacidez moderada no abdome superior.

\section{Pacientes e metodo}

Foram operadas 58 pacientes no período de 1994 a 2007, todas do sexo feminino, com idade média de 41 anos, variando entre 23 e 52 anos, todas com anestesia peridural. A técnica empregada foi a combinação de procedimentos consagrados na literatura, sendo marcada previamente a porção de pele a ser ressecada no abdome inferior. Inicialmente, houve lipoaspiração do dorso, de cintura e de toda a região anterior do abdome. $\mathrm{Na}$ área central supra-umbilical, foi aspirado no plano superficial e profundo, a fim de definir os músculos retos abdominais. No restante do abdome, a lipoaspiração foi profunda, a fim de tentar preservar a irrigação da pele, uma vez que houve descolamento em área considerável (Fig. 1). Após lipoaspiração (Quadro 1), foi realizada incisão baixa na região pubiana, variando entre 13 e $18 \mathrm{~cm}$ (Quadro 2). Descolou-se em toda a região a ser ressecada, sendo então direcionado superiormente para a região mediana do abdome até aproximadamente $5 \mathrm{~cm}$ da borda lateral

Quadro1. Volume aspirado durante a lipoabdominoplastia:

\begin{tabular}{lr} 
Volume & n \\
1000 a $1500 \mathrm{ml}$ & 22 \\
1500 a $2000 \mathrm{ml}$ & 25 \\
2000 a $2200 \mathrm{ml}$ & 11 \\
Total & 58 \\
\hline
\end{tabular}

Quadro 2. Extensão da insisão para a realização da lipoabdominoplastia:

\begin{tabular}{lr} 
Tamanho & $\mathbf{n}$ \\
13 a $14 \mathrm{~cm}$ & 22 \\
14 a $15 \mathrm{~cm}$ & 25 \\
15 a $16 \mathrm{~cm}$ & 07 \\
16 a $17 \mathrm{~cm}$ & 03 \\
17 a $18 \mathrm{~cm}$ & 01 \\
Total & 58 \\
\hline
\end{tabular}

Quadro 3. Altura de reinserção do umbigo após ressecção do excesso de pele:

\begin{tabular}{lr} 
Reinserção do umbigo & n \\
$3 \mathrm{~cm}$ mais baixo & 39 \\
$4 \mathrm{~cm}$ mais baixo & 17 \\
$5 \mathrm{~cm}$ mais baixo & 02 \\
Total & 58 \\
\hline
\end{tabular}

Quadro 4. Extensão de ressecção de excesso de pele após descolamento do retalho abdominal (sem aplicar tração):

\begin{tabular}{lr} 
Ressecção de pele & n \\
8 a $9 \mathrm{~cm}$ & 32 \\
9 a $10 \mathrm{~cm}$ & 18 \\
10 a $11 \mathrm{~cm}$ & 08 \\
Total & 58 \\
\hline
\end{tabular}

Quadro 5. Distribuição pré-operatória das características do abdômen das pacientes:

\begin{tabular}{ll} 
Características do abdômen & n \\
$\begin{array}{ll}\text { Aumento do volume total e flacidez moderada } \\
\text { do abdômen superior }\end{array}$ & 48 \\
Aumento de volume e flacidez mais acentuada & \\
do abdômen superior & 02 \\
Abdominoplastia clássica prévia & 03 \\
Miniabdominoplastia prévia & 05 \\
Total & 58 \\
\hline
\end{tabular}

Quadro 6. Complicações:

Complicações

Presença de alguma flacidez

no abdome superior

Cicatriz alargada

Ondulações na área aspirada

Total 
dos retos. O umbigo foi seccionado na base e permaneceu preso ao retalho abdominal (Fig. 1). Isso permitia que fosse corrigida uma eventual diastase com plicatura dos retos abdominais desde o apêndice xifóide até o pubis (Fig. 2). Após plicatura, reinseriu-se o umbigo na aponeurose aproximadamente 2 a $5 \mathrm{~cm}$ abaixo da posição original, a fim de tratar também alguma flacidez de pele do abdome superior (Quadro 3). Utilizando pontos de nylon 3-0 configurou-se facilmente a largura, o tamanho e a profundidade do novo umbigo. Excepcionalmente, nos casos de maior flacidez no abdome superior e umbigo alto, reimplantou-se o umbigo até $5 \mathrm{~cm}$ mais baixo (Fig. 2). Em seguida passou-se à ressecção do excesso de pele previamente marcado, ampliando-se além da marcação nos casos de maior flacidez após a lipoaspiração e o descolamento. A porção de pele ressecada do abdome inferior variou entre 8 e $11 \mathrm{~cm}$ (Quadro 4) (Fig. 3). Fechou-se a ferida cirúrgica com sutura intradérmica, com drenagem sob aspiração e pressoterapia com cinta elástica por 2 meses.

\section{Resultados}

No período de 1994 a 2007, foram operadas 58 pacientes do sexo feminino, com idade média de 41 anos, variando entre 23 e 52 anos, todas com anestesia peridural. Aumento do volume abdominal e flacidez moderada do abdomen superior foi observado em 48 casos. Em 2 casos havia flacidez mais acentuada e 3 pacientes já haviam sido submetidas a abdominoplastia clássica, que, apesar do resultado satisfatório no abdome inferior, apresentavam alguma flacidez e excesso de volume adiposo no abdômen superior. Cinco pacientes já haviam sido submetidas a miniabdominoplastia, permanecendo com flacidez e volume no abdome superior (Quadro 5). Todas foram operadas com a mesma combinação de procedimentos cirúrgicos descritos no presente trabalho.

Os resultados foram satisfatórios nos casos de aumento do volume abdominal e flacidez no abdome inferior com moderada flacidez do abdome superior. Em duas pacientes a flacidez do abdome superior já sugeria indicação de abdominoplastia completa, mas as pacientes pretendiam engravidar e não aceitaram tal indicação. Nesses casos, permaneceu alguma flacidez superior. No restante, as complicações foram pequenas irregularidades e discretas alterações cicatriciais, sendo corrigidas posteriormente com procedimentos ambulatoriais (Quadro 6) (Fig. 4, 6).

\section{Discussao}

As alterações estéticas da região abdominal são facilmente corrigidas com lipoaspiração isolada quando não há flacidez abdominal. A aborda- gem é simples em casos de grande volume e muita flacidez através da abdominoplastia clássica, ou miniabdominoplastia convencional quando há deformidade restrita à região infraumbilical (1,3-5). Entretanto, a solução em casos de flacidez moderada no abdome superior ou umbigo excessivamente alto se torna mais complicada, sendo necessárias medidas não convencionais.

A abordagem dessas alterações estéticas é assunto amplamente estudado. Hackme (6) publicou detalhado estudo sobre evolução de técnicas para correção de excessos abdominais. Já o tratamento de aumento do volume adiposo em pacientes sem flacidez de pele teve nos trabalhos de Illouz (7) grande evolução, com a lipoaspiração. Callia (5) estudou minuciosamente o tratamento de abdome pendular com grande volume, enquanto Wilkinson e Swartz (9) e Greminger (10) introduziram importantes conceitos no tratamento do abdome inferior.

A cicatriz umbilical também foi bastante estudada, e abordagens externas com desenhos variados foram descritas, mas a liberação do umbigo na implantação aponeurótica e posterior reimplante foi descrita por Callia (8) e Avelar (11), que realizava sua reimplantação cerca de 2 $\mathrm{cm}$ mais baixo.

Após utilização ampla da lipoaspiração, diversos estudos demonstraram a possibilidade do uso associado à abdominoplastia. Matarasso(3,4) utiliza a lipoaspiração em todos os tipos de abdominoplastia, restringindo seu uso mais à área não descolada. Valiosos estudos anatômicos como os de Huger (12) permitiram que fossem realizados procedimentos cada vez mais seguros nas abdominoplastias. O descolamento limitado torna mais seguro o procedimento, pois preserva a vascularização do retalho $(4,13)$. Saldanha (13) e Graf (5) defendem que a lipoaspiraçao da região supraumbilical pode trazer comprometimento ao retalho, mas não houve sinal de sofrimento dessa área.

\section{Conclussao}

A tecnica empregada é válida, pois permite o tratamento da flacidez moderada do abdome superior e inferior com incisão de tamanho aceitável. O descolamento reduzido torna viável a realização da lipoaspiração de volumeconsiderável. A ausência de cicatriz externa umbilical evita o estigma, deixando o umbigo com aspecto estético natural e as complicações no geral são relativamente raras, semelhantes às encontradas nas abdominoplastias convencionais. 


\title{
Comentario al trabajo ilipoabdominoplastia con desinserción umbilical y despegamiento ahdominal reducidon
}

\author{
Dr. Carlos del Pino Roxo \\ J efe del Servicio de Cirugía Plástica del Hospital General Andarí, Río de J aneiro, Brasil \\ Chefe do Serviço de Cirurgia Plástica, Hospital General Andarí, Río do J aneiro. Brazil
}

Observando el trabajo podemos notar que se trata de un procedimiento que requiere mucha atención por parte del cirujano, dado que se trata de un abordaje que nos permite apenas corregir pequeños excesos de piel de la región supraumbilical, y que tiene en nuestra opinión una buena indicación para la resección del exceso de piel en la región infraumbilical.

Pensamos que, el obligo solo debe ser cambiado de su posición original en aquellas pacientes en las que se encuentre más arriba de lo normal, y que la colocación del mismo en un punto más bajo puede provocar una gran insatisfacción y ser además muy difícil de corregir.

En cuanto a la asociación de lipoaspiración superficial y profunda en todo el abdomen, con despegamiento del colgajo abdominal, creemos que dicho despegamiento debe ser moderado pues la posibilidad de un sufrimiento de dicho colgajo es muy grande, y no creemos que sea posible un buen resultado de abdominoplastia sin un tratamiento de la pared abdominal (plicatura), lo que conlleva la necesidad de disecar hasta el apéndice xifoides.

Para terminar, creemos que una cicatriz bien posicionada queda mejor aun siendo más grande que una no tan bien posicionada, pues permite a las pacientes usar trajes de baño y ropa interior más sensuales.

Espero con este comentario acrecentar y no disminuir las cualidades del trabajo presentado.
Observando-se o trabalho em tela, podemos notar que se trata de um procedimento que requer muita atenção por parte do cirurgião uma vez que esta abordagem nos permite apenas corrigir pequenos excessos de pele na região supra umbelical, tendo em nossa opinião boa indicação para ressecção de excesso de pele em região infra umbelical.

È nossa opinião, que a cicatriz umbelical deve ser alterada de sua posição apenas em pacientes nos quais se encontre mais acima do que o normal,e que a colocação da mesma em um ponto mais baixo pode trazer uma insatisfação muito grande às pacientes, sendo a mesma de difícil correção.

Quanto à associação de lipoaspiração superficial e profunda,de todo abdomem,com o descolamento do retalho abdominal, cremos que tal descolamento deva ser bastante moderado afim de evitar-mos a ocorrencia de sofrimento do retalho abdominal,cujapossibilidade é bem grande, uma vez que não acreditamos em bom resultado de abdominoplastia sem o tratamento da parede abdominal(plicatura), o que nos traz a nescessidade de dissecçao até o apendice xifóide.

Para terminarmos temos a acrescentar que mais importante que o tamanho de uma cicatriz em abdominoplastia, é a sua localização, pois uma cicatriz maior bem posicionada fica muito melhor que uma cicatriz menor mal posicionada, pois permite o uso de biquinis e roupas mais sensuais.

Espero que estas considerações acrescentem, e não diminuam qualidade ao trabalho apresentado.

\section{Respuesta al comentario del Dr. Carlos del Pino Roxo}

\section{Dr. Cido Carvalho}

En relación con el comentario del Dr. Roxo, debo aclarar que la indicación se restringe a aquellas pacientes con flacidez moderada del abdomen superior, y la lipoaspiración se hace en el plano profundo en toda la zona despegada, y solo en la línea media supra e infraumbilical, la lipoaspiración es superficial y profunda; el despegamiento se hace hasta apéndice xifoides, lo que permite una plicatura de los rectos igual a la de una plicatura convencional.
Em relação ao comentário do Dr. Roxo, devo esclarecer que a indicação é restrita à paciente com flacidez moderada no abdome superior; a lipoaspiração é somente na camada profunda, em toda a área descolada, e que apenas na linha média supra-umbilical é que a lipoaspiração é superficial e profunda; o descolamento é feito até o apêndice xifóide, permitindo a plicatura dos retos como na abdominoplastia convencional. 